Gut, 1960, 1, 366.

\title{
RADIOLOGICAL INVESTIGATION OF THE SMALL INTESTINE BY SMALL BOWEL ENEMA TECHNIQUE
}

BY

\author{
F. PYGOTT, D. F. STREET, M. F. SHELlSHEAR, and C. J. RHODES
}

From the Central Middlesex Hospital, London

This paper records some experience with the Scott Harden technique of small intestinal intubation for performing small bowel enemas. It is particularly recommended for searching for obstructive or infiltrative lesions of the small intestine.

A completely satisfactory demonstration of the entire small bowel by the usual barium followthrough series, or one of its many modifications, is often not attained in the normal subject, and difficulties are intensified in the presence of pathology. Individual loops of bowel may fill and empty rapidly, and, however short the intervals between examinations, these loops may not be shown effectively. Gross delay in the onward passage of barium may frustrate the efforts of the radiologist to show the exact site and probable nature of an obstructing lesion, or several intestinal loops may overlap and so obscure a lesion; this is particularly true of ileal loops lying in the pelvis. Since the radiologist is unable to affect the degree of filling attained in the region under immediate scrutiny, the significance of local constrictions or dilatations observed at any one examination in the series is obscure and it may not be possible to detect the changes later. Local destructive processes in the mucous membrane may be quite unrecognizable.

Dissatisfaction with the barium follow-through examination led Schatzki (1943) to adopt the small bowel enema technique of duodenal intubation, using a small rubber tube and controlled filling of the small intestine, that had been recommended earlier by Pesquera (1929). The disadvantages of this method are the uncertain delay during which the tube is awaiting its passage from the stomach into the duodenum and the high failure rate because the tube cannot be passed in reasonable time. Immediate transgastric intubation with a flexible polythene tube does not impose delay and the success rate is much higher. The technique that we have used is essentially that described by Scott Harden (1960) with minor modifications, though some early cases were examined by a single tube method.
Description of THE Method

The equipment required consists of a flexible, but semi-rigid, polythene tube, $4 \mathrm{~mm}$. inside diameter $(5.8 \mathrm{~mm}$. outside diameter) about 1 metre long, through which a second polythene tube of $1.5 \mathrm{~mm}$. inside diameter, $\left(2.7 \mathrm{~mm}\right.$. outside diameter), about $1 \frac{1}{2}$ metres long, can slide easily. The distal end of each tube has a small brass ring or ferrule bonded to it so that its position can be identified radiologically. We have found that a short, tightly fitting 3- or 4-in. length of flexible polythene tube sheathed over the distal end of the outer semi-rigid tube so that it projects 2 or 3 in. beyond the tip is an advantage. The tubing is sterilized by boiling and care is taken to avoid coiling or pinching the tubes in removing them from the sterilizer. A connexion is arranged with the proximal end of the inner tube so that a suspension of "micropaque", $1: 1$ part of tap water, can be injected through it from a $50 \mathrm{ml}$. syringe.

The patient is prepared as for a barium meal examination. The object of the examination and the method are clearly explained, Nearly all patients have had a barium meal beforehand and abnormalities of the stomach and duodenal cap should be excluded before this technique is employed. The patient is then given a tablet of amethocaine to suck and the fauces are lightly sprayed with $2 \%$ xylocaine.

The tubing has been carefully examined beforehand for patency and free movement of the inner on the outer is assured. The inner tube is passed down the outer until its distal end is just short of that of the outer. An assistant holds the tubing while the radiologist passes the distal end gently through the patient's mouth over the epiglottis into the hypopharynx. The tube should progress easily with gentle pressure once the cricopharyngeus has been passed. Progress is continued until a sense of elastic resistance is felt, due to the tube impacting against the greater curve of the stomach. The tube is then withdrawn slightly and the patient is placed supine on the $x$-ray couch. We use a Marconi type 5-in. image amplifier for the examination, and take under and over 
couch large films as required. (The actual screening factors used are $90-95 \mathrm{kV}$. with $0.3 \mathrm{~mA}$.) When radiological examination has identified the position of the lower ends of the tubes, then the inner tube is carefully advanced through the outer until it engages the pylorus and is slipped through it as far as possible. Coiling of the inner tube within the stomach can often be avoided by rotating the patient to the right and by carefully advancing the outer tube over the inner immediately the latter shows any tendency to turn upwards. In a very few cases the duodenum cannot be intubated in spite of every effort, usually in grossly distorted stomachs with a severe cup and spill shape. As with all practical procedures increased experience brings increased facility and fewer failures. In the $\mathbf{2 1}$ months we have been using the technique we have attempted 73 examinations with four complete failures, one more patient refused all cooperation before trying, and in one more intubation was effected but the examination was not entirely satisfactory. The 67 patients in whom the examination was satisfactory give a success rate of, at worst, $90 \%$, and this may well be improved upon.

When the tip of the inner tube has been introduced into the duodenum a little of the barium suspension is steadily injected under radiological control. Back flow into the stomach does not normally occur even if the tip is only in the duodenal cap. An early film is taken to show the duodenal loop and the uppermost loops of jejunum filled. Successive injections of barium suspension and tap water are made as required and the progress of the contrast medium observed, controlled filling of the intestine is attainable, and in the normal subject the caecum is reached in 15 to 20 minutes. In the abnormal case irregularities in the lumen can be examined continuously with different degrees of distension and the area can be re-examined by washing it through with water and refilling with barium.

Delay in forward flow indicates the presence of an obstructive lesion ahead and discretion must be used in avoiding proximal overfilling; nevertheless, with patience it is nearly always possible to identify the exact site of any kinking or narrowing of the lumen in a way that is rarely possible during a follow-through examination. In infiltrative lesions, such as Crohn's disease, it is possible to form a much more accurate impression of the extent of involvement as the rigidity of the unconstricted bowel can be assessed because its tubular stiffening can be recognized since filling can be made complete. Also local areas of narrowing can be recognized and their existence confirmed.

\section{Illustrative CASES}

Figs. 1 and 2 show the appearance of the small bowel in a normal subject, as demonstrated by this method.

CASE 1.-A woman aged 29 years gave a history of 11 years' diarrhoea, the bowels opening seven to 20 times daily, and periodic pain in the right iliac fossa. The onset of symptoms followed appendicectomy for pain in the right iliac fossa. Her stools were watery, green at times, and less often had blood in them. On examination a small mass was felt in the right iliac fossa.

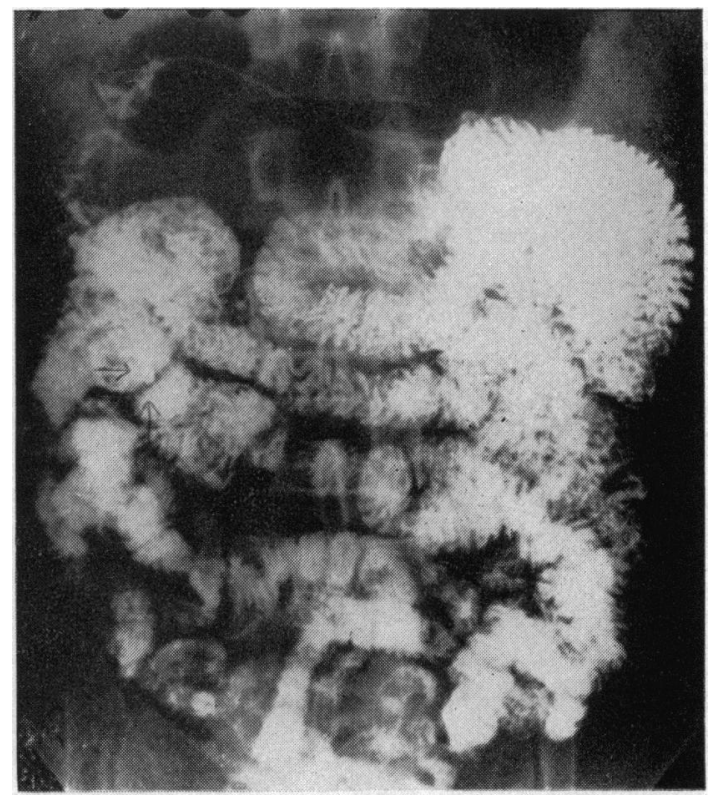

FIG. 1.-Normal appearances of jejunum and upper ileum with small bowel enema technique. The position of the lower end of the inner tube is arrowed, the lower end of the outer is at the cardia.

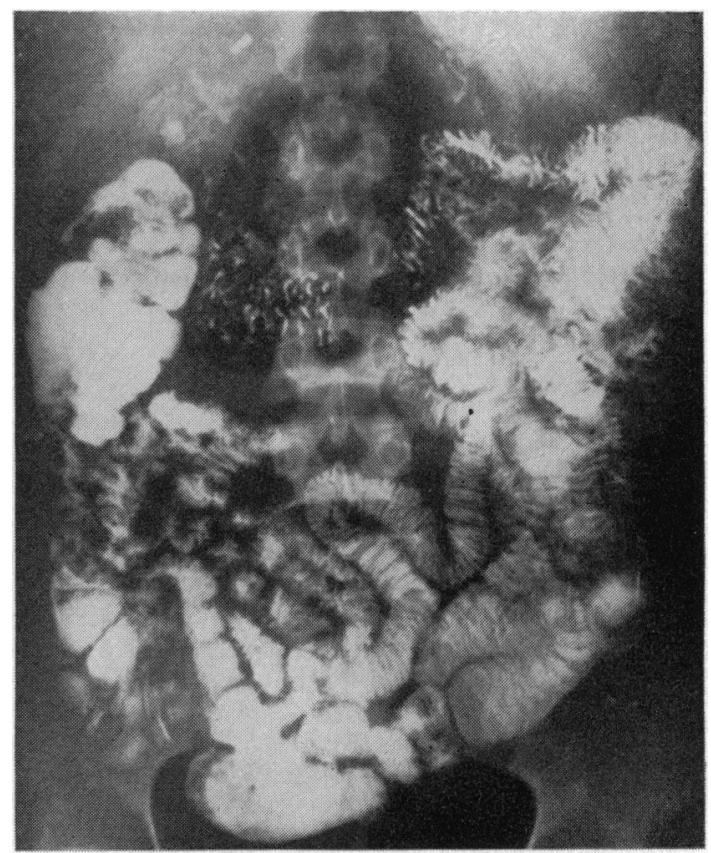

FIG. 2.-The whole of the small bowel, except the duodenal loop, is shown. The proximal colon is filled. The lower end of the inner tube is in the duodenal cap; there is some back leak into the stomach. 


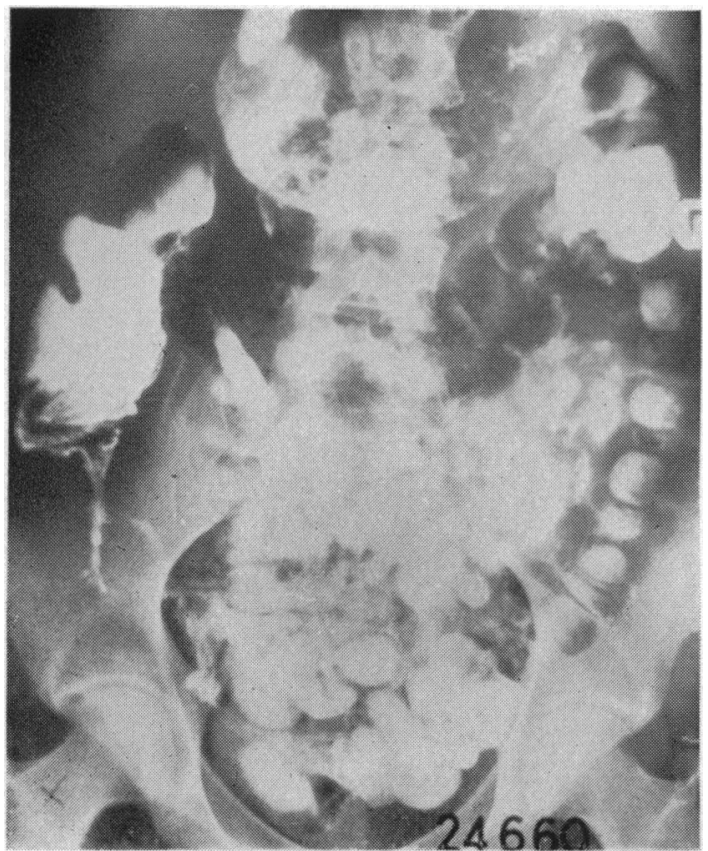

Fig. 3.-Film showing extensive Crohn's disease in the lower ileum, caecum, hepatic flexure, and proximal transverse colon.

A small bowel enema showed a constrictive lesion in the lower ileum, several inches long, and shorter lesions in the caecum, hepatic flexure, and transverse colon (Fig. 3).

Operation by Mr. J. W. P. Gummer confirmed these findings and he removed $2 \mathrm{ft}$. of distal ileum and the right half of the colon. Histology showed typical Crohn's disease.

CASE 2.-A woman aged 23 years gave a history of continuous bowel irregularity and diarrhoea since the age of 9 months. For seven years she had had bouts of colicky abdominal pain. At the age of 14 radiographs had shown "distended gut". Recently, symptoms had become more severe and she was incontinent on account of the diarrhoea. Her weight was under 5 stones. Clinical examination revealed abdominal distension but no mass. She had a hypokalaemia $(2.8 \mathrm{mEq}$./1.). A barium meal examination, performed at another hospital six months before her admission, was reported to show "atony of the terminal ileum". Numerous calcified glands were shown in the right iliac fossa.

A small bowel enema (Fig. 4) showed gross distension of the terminal loop of ileum with lesser distension proximal to this. There was gross constriction of the whole of the caecum and ascending colon. The constriction terminated abruptly at the ileo-caecal valve.

Laparotomy by Mr. Gummer showed a dense fibrous mass involving the whole of the caecum and ascending colon with gross proximal distension of the ileum. A right psoas abscess was present. (There was no radiological evidence of lumbar or dorsal spine disease). Many calcified and partially calcified glands were present. The distal ileum and proximal colon were resected with great difficulty, and the descending duodenum was unavoidably opened in separating the ascending colon from it. Unfortunately, the patient died eight days later.

Necropsy confirmed the absence of any focus of disease in the spine and the psoas abscess was due to direct extension from the caecal lesion. Histology of the resected specimen showed dense fibrotic changes but no evidence of any active tuberculous lesion; granulomatous and partially calcified tuberculous disease was present in the lymph glands.

CASE 3.-A man aged 25 years gave a history of abdominal pains and diarrhoea which began six years before while he was at sea. Other members of the crew had had similar trouble at the time but their symptoms cleared up and his did not. The attacks became more frequent with the passage of time though his general health was maintained. Clinical examination showed no

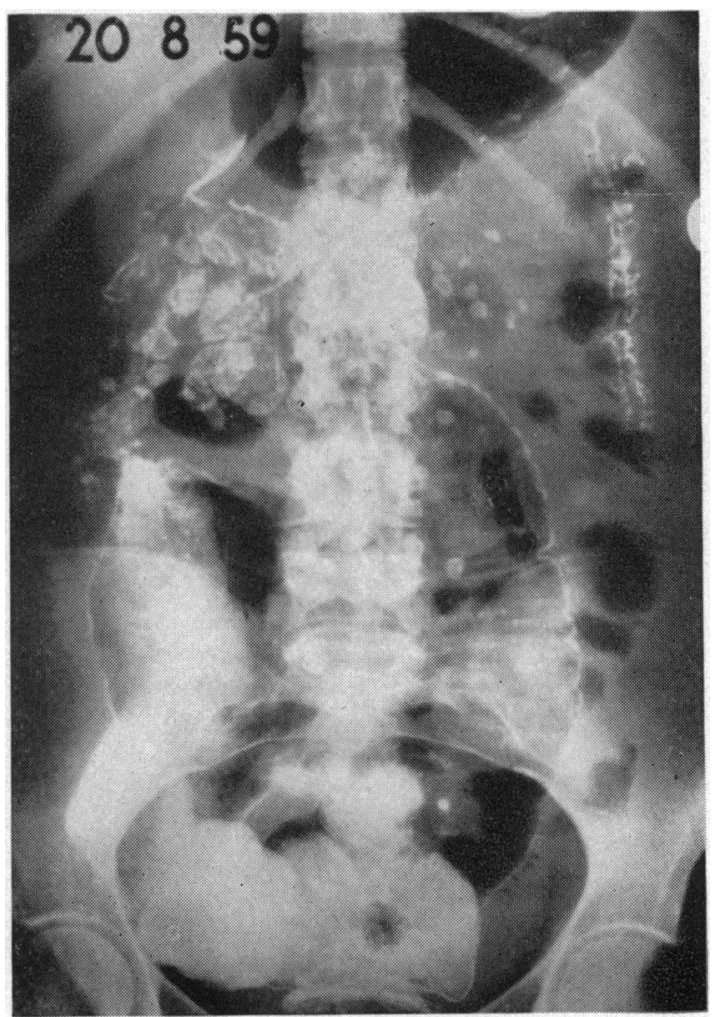

Fig. 4.-Late film showing a very dilated lowermost loop of ileum. The caecum is grossly contracted and almost entirely obscured by the large calcified glandular mass in the right iliac fossa. Fibrous contracture of caecum and ascending colon is probably due to old tuberculous disease. 


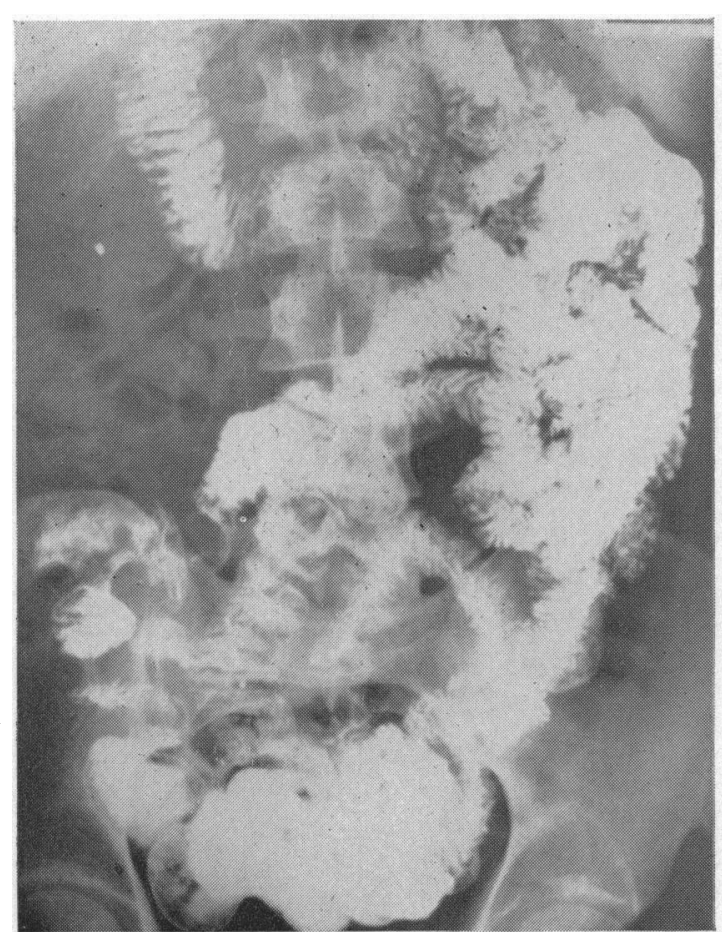

FIGS. 5 AND 6.-Film (single tube method) showing multiple strictures in lower ileum. Histologically, necrotizing ileitis or? Crohn's disease. Fig. 5 shows the normality of the upper small bowel, and Fig. 6 five or six of the strictures.

abnormality except for tenderness in the right iliac fossa. Repeated barium meals had shown some duodenal cap deformity. A small bowel enema (Figs. 5 and 6) showed numerous very localized constrictions in the lower ileum with intervening normal bowel. The mucosal pattern was locally destroyed. Laparotomy by Mr. Gummer showed numerous areas of stricture in the lower ileum associated with regional glandular enlargement. One of the areas was excised and histology revealed simple mucosal ulceration with necrotic areas and granulations. Dr. R. Drury reported that the appearances, though not typical, could be due to Crohn's disease. Ultimately, $4 \mathrm{ft}$. of ileum had to be resected and a right hemicolectomy be performed at a subsequent operation after a spontaneous perforation at one of the sites of constriction.

\section{Applications of the Method}

Success in this investigation depends on full cooperation on the part of the patient, who must be sufficiently ambulant to be able to submit to a somewhat rigorous examination. Many will have had one or several barium meal examinations and we like to have the results of these available. Abnormalities in the stomach and duodenal cap must

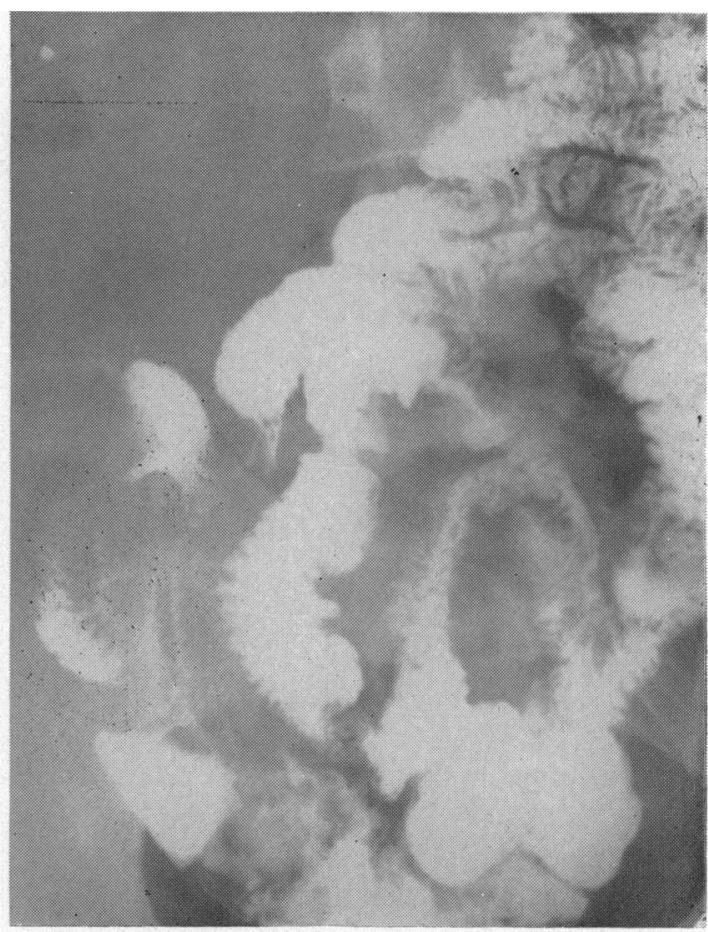

have been excluded and films showing the outline of the stomach help in indicating the probable ease or difficulty of intubation.

Since the examination is more anatomical than physiological in character we prefer not to employ it as a primary examination in patients presenting with steatorrhoea, though we do not hesitate to use it secondarily in such patients. In spite of what has just been said, characteristic steatorrhoea-type appearances are produced when the method is applied to patients with an idiopathic or pancreatic steatorrhoea.

The method is very valuable in patients presenting with abdominal pain and diarrhoea in whom an obstructive or constrictive lesion of the small bowel is likely to be found. In patients who are known to have Crohn's disease and who have had one or more operations, sometimes of unknown nature, it is most helpful in giving the surgeon as accurate an impression as possible of the state of affairs he may have to face before he undertakes a difficult laparotomy. The presence of "blind loops" may be more than suspected. In investigating the small bowel for the source of occult bleeding it is, in our 
opinion, the most effective radiological method.

In conclusion, we recommend this investigation when it can be used with care and discretion in the search for obstructive or infiltrative lesions of the small bowel or where occult bleeding is likely to be coming from a lesion below the duodenal cap. We would stress that it is not to be undertaken as a routine but its application is most advantageous in the circumstances indicated.
We are obliged to our clinical colleagues, especially Dr. F. Avery Jones and Mr. J. W. P. Gummer, for access to their findings and to Dr. R. Drury for his pathological reports on the cases quoted.

\section{REFERENCES}

Harden, W. G. Scott (1960). In Modern Trends in Diagnostic Radiology, (Third Series), ed. Dr. J. W. McLaren, p. 84. Butterworths, London.

Pesquera, G. S. (1929). Amer. J. Roentgenol, 22, 254.

Schatzki, R. (1943). Ibid., 50, 743. 